\title{
c-Jun/Ap-1 is upregulated in an Ang II-induced abdominal aortic aneurysm formation model and mediates Chop expression in mouse aortic smooth muscle cells
}

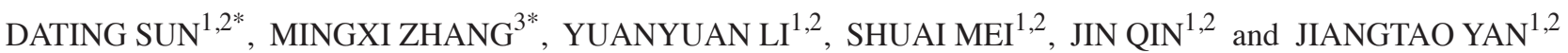 \\ ${ }^{1}$ Division of Cardiology, Department of Internal Medicine, Tongji Hospital, Tongji Medical College, \\ Huazhong University of Science and Technology; ${ }^{2}$ Hubei Key Laboratory of Genetics and Molecular \\ Mechanisms of Cardiological Disorders; ${ }^{3}$ Division of Cardiology, Department of Internal Medicine, \\ Wuhan Hospital of Traditional Chinese Medicine, Wuhan, Hubei 430030, P.R. China
}

Received July 14, 2018; Accepted February 8, 2019

DOI: $10.3892 / \mathrm{mmr} .2019 .10017$

\begin{abstract}
Abdominal aortic aneurysm (AAA) is an asymptomatic, potentially lethal disease whose ruptures have a high mortality rate. An effective pharmacological approach to decrease expansion or prevent the rupture of AAAs in humans remains lacking. Previous studies have suggested that activator protein 1 (c-Jun/AP-1) and C/EBP homologous protein (Chop) are involved in the development of AAA. The purpose of the present study was to investigate whether c-Jun/AP-1 mediates Chop overexpression in AAA. c-Jun/AP-1 and Chop protein levels were determined in an angiotensin II (Ang II)-induced AAA model using apolipoprotein E-deficient mice. Additionally, mouse aortic smooth muscle cells (MOVAS cell line) were treated with Ang II. Apoptosis was evaluated via TUNEL assay, MOVAS cell migration ability was assessed by monolayer wound healing assay and the levels of c-Jun/AP-1 and Chop were determined by western blotting, immunofluorescence and immunocytochemical assays. Following c-Jun silencing using c-Jun-specific small interfering (si)RNA, Chop expression was evaluated. Furthermore, chromatin immunoprecipitation (ChIP) was used to investigate whether c-Jun/Ap-1 binds directly to the DNA damage-inducible transcript 3 protein (Ddit3) promoter. It was observed that
\end{abstract}

Correspondence to: Dr Jiangtao Yan, Division of Cardiology, Department of Internal Medicine, Tongji Hospital, Tongji Medical College, Huazhong University of Science and Technology, 1095 Jiefang Avenue, Wuhan, Hubei 430030, P.R. China

E-mail: jtyan_tjh@163.com

*Contributed equally

Abbreviations: AAA, abdominal aortic aneurysm; Ang II, angiotensin II; Ap-1, activator protein-1; Chop, C/EBP homologous protein; ApoE-/-, apolipoprotein E-deficient; ChIP, chromatin immunoprecipitation

Key words: AAA, Ang II, transcription factors, c-Jun/AP-1, Chop
c-Jun/AP-1 and Chop were synchronously overexpressed in Ang II-induced AAA and Ang II-treated cells, and that apoptosis and migration were induced by Ang II. In addition, Chop was suppressed when c-Jun was silenced by targeted siRNA. Notably, the ChIP assay demonstrated that the DNA fragments pulled down by primary antibodies against c-Jun/Ap-1 were able to be amplified by (Ddit3) promoter-specific primers. c-Jun/AP-1 may therefore mediate Chop expression in MOVAS cells via Ddit3. These results suggested that c-Jun/AP-1 may be a novel target for AAA therapy.

\section{Introduction}

Abdominal aortic aneurysm (AAA) involves chronic transmural inflammation and structural deterioration of the tissue architecture, leading to a progressively enlarged abdominal aorta. AAAs usually remain asymptomatic until a rupture occurs, which is associated with high morbidity and mortality rates in the adult human population $(1,2)$, particularly in male patients over the age of 65 years (3). Using an AAA mouse model, it has been previously demonstrated that statins exert beneficial effects on AAA progression by inhibiting endoplasmic reticulum (ER) stress and C/EBP homologous protein (Chop) expression (4). Another study demonstrated that the selective inhibition of c-Jun $\mathrm{N}$-terminal kinase (JNK), also known as stress-activated protein kinase, not only prevented the development of AAA, but also caused the regression of established AAA (5). Angiotensin-converting enzyme inhibitors and angiotensin II receptor blockers (ARBs) may also reduce AAA mortality (6), but to the best of our knowledge, there are no pharmacological methods that are able to efficiently decrease the expansion or prevent the rupture of an AAA in humans (7).

Activator protein 1 (AP-1) is a dimeric transcription factor that controls gene expression, cell proliferation, differentiation and apoptosis $(8,9)$. AP-1 consists of Jun (c-Jun, JunB and JunD) and Fos (c-Fos, FosB, Fra1 and Fra2) family members. AP-1 proteins regulate target gene expression by binding to specific DNA sequences $(10,11)$. Jun proteins are able to homo- and heterodimerize, whereas Fos proteins are unable 
to homodimerize, but heterodimerize with Jun proteins for DNA binding. Jun-Fos heterodimers bind preferentially to a heptamer consensus sequence known as the TPA response element (12). Activated JNK upregulates c-Jun and facilitates c-Jun phosphorylation at ser63 and ser73. Additionally, activated c-Jun is involved in the formation of AP-1 (13). Angiotensin II (Ang II), a small peptide and principal component of the renin-angiotensin system (14), may also activate AP-1 by stimulating reactive oxygen species generation and activating the JNK pathway $(14,15)$.

C/EBP homologous protein (Chop), also termed GADD153, is a transcription factor and a member of the basic leucine zipper domain family (16), encoded by DNA damage-inducible transcript 3 protein (Ddit3). Chop may be activated at multiple levels during ER stress (17) and is commonly used as an ER stress indicator. Chop overexpression promotes apoptosis in several cell lines $(18,19)$. It has previously been demonstrated that Chop is overexpressed in an Ang II-induced AAA mouse model (4). Another study revealed that Chop may not only act as a regulator of $\mathrm{C} / \mathrm{EBP}$ target genes, but may also be tethered to AP-1 and thereby activate AP-1 target genes (20).

In the present study, the molecular mechanisms underlying AAA development were evaluated with a focus on the roles of c-Jun/AP-1 and Chop, and it was determined that c-Jun/AP-1 is overexpressed in an Ang II-induced AAA model and Ang II-treated mouse aortic smooth muscle cell line (MOVAS cells). Chop was synchronously overexpressed; the regulation of Chop expression and apoptosis by c-Jun/AP-1 in AAA was then investigated. c-Jun/AP-1 may have had an essential role as a transcriptional regulator of Ddit3, resulting in Chop overexpression and the acceleration of AAA development.

\section{Materials and methods}

Animals and AAA models. All animals were housed at the animal care facility of Tongji Medical College, (Wuhan, China) under specific pathogen-free conditions and were fed a normal diet. Mice were housed in temperature-controlled cages $\left(20 \pm 1^{\circ} \mathrm{C}, 55 \pm 5 \%\right.$ humidity) with a $12 \mathrm{~h}$ light-dark cycle and given free access to water and food. All animal experiments were performed in accordance with the Animal Research Reporting of in vivo Experiments and the National Institutes of Health guidelines for animal welfare (21), and the study was approved by the Animal Research Committee of Tongji Medical College.

A total of 30 10-week-old apolipoprotein E-deficient (ApoE ${ }^{-/}$; C57BL/6 background) male mice ( 20 g) were purchased from Beijing Huafukang Biotechnology Co., Ltd. (Beijing, China). Mice were randomly divided into 2 groups ( $n=15$ per group) and implanted with osmotic pumps (Model 2004; Durect Corporation, Cupertino, CA, USA) containing either Ang II (1,000 ng/kg/min; Sigma-Aldrich; Merck KGaA, Darmstadt, Germany) or saline (sham) for 4 weeks (22). Mice were anesthetized with isoflurane using an anesthesia machine prior to surgery. After 4 weeks, animal aortic tissues and blood samples were obtained following sacrifice, and samples were stored at $-80^{\circ} \mathrm{C}$ for western blotting and immunofluorescence.

Histological analysis. Aortas were fixed in $4 \%$ paraformaldehyde dissolved in PBS at room temperature for $24 \mathrm{~h}$ and embedded in paraffin for histological analyses. Serial cross-sections $(3 \mu \mathrm{m})$ were produced from the aorta. For the morphometric analysis, the sections were stained with hematoxylin and eosin at room temperature. Briefly, the sections were incubated with hematoxylin for 5-10 min following rehydration, washed with $1 \%$ ethanol hydrochloride for $5 \mathrm{sec}$, and stained with eosin for $3 \mathrm{~min}$.

Immunofluorescence staining. The aorta specimens were prepared as described above. The arterial sections were blocked in PBS with $10 \%$ fetal bovine serum (FBS; Gibco; Thermo Fisher Scientific, Inc., Waltham, MA, USA) at room temperature for $1 \mathrm{~h}$, and incubated overnight at $4^{\circ} \mathrm{C}$ with primary antibodies against c-Jun/Ap-1 (cat. no. 711202; dilution, 1:200; Invitrogen; Thermo Fisher Scientific, Inc.) and $\alpha$-smooth muscle actin ( $\alpha$-SMA; cat. no. BM0002; dilution, 1:50; Boster Biological Technology, Pleasanton, CA, USA). Antibodies were replaced with secondary antibodies labelled with Alexa Fluor $^{\circledR}$ 568-conjugated goat anti-rabbit Immunoglobulin G (IgG; cat. no. A-11034; dilution, 1:250; Invitrogen; Thermo Fisher Scientific, Inc.) and Alexa Fluor ${ }^{\circledR} 488$-conjugated goat anti-mouse IgG (cat. no. A32727; dilution, 1:250; Invitrogen; Thermo Fisher Scientific, Inc.). Cell nuclei were stained with DAPI at room temperature for $20 \mathrm{~min}$. Images were obtained using an E2000U confocal microscope (magnification, x200 and x400; Nikon Corporation, Tokyo, Japan) and were merged using Image Pro Plus (version 6.0; Media Cybernetics, Rockville, MD, USA).

Cell culture and treatment. MOVAS cells were purchased from the American Type Culture Collection (Manassas, VA, USA) and were cultured in Dulbecco's Modified Eagle's medium (DMEM) containing 10\% FBS (cat. no. 10099-141; Gibco; Thermo Fisher Scientific, Inc.) in a humidified atmosphere at $37^{\circ} \mathrm{C}$ with $5 \% \mathrm{CO}_{2}$. MOVAS cells were treated with various concentrations of Ang II (1, 10, 20, 50 and $100 \mathrm{nM})$ for $24 \mathrm{~h}$. c-Jun small interfering RNA (siRNA), designed and synthesized by Guangzhou RiboBio Co., Ltd. (Guangzhou, China), was transfected at a final concentration of $50 \mathrm{nM}$ using Lipofectamine $^{\circledR} 2000$ (cat. no. 11668019; Invitrogen; Thermo Fisher Scientific, Inc.) for 24 or $48 \mathrm{~h}$ prior to subsequent experimentation, following the manufacturer's protocol. The c-Jun-si1 target sequence was 5'-GCCAACTCATGCTAA CGCA-3', the c-Jun-si2 target sequence was 5'-CAGCTTCCT GCCTTTGTAA-3', and the c-Jun-si3 target sequence was 5'-GCGCATGAGGAACCGCATT-3'.

Immunocytochemistry and confocal imaging. MOVAS cells $\left(4 \times 10^{5}\right.$ cells/well) were plated in 6 -well glass slide chambers (Iwaki Glass, Tokyo, Japan) and treated with (or without) Ang II (20 nM) for $36 \mathrm{~h}$. Cells were washed with PBS twice and fixed in $4 \%$ paraformaldehyde for $15 \mathrm{~min}$ at room temperature. Following washing with PBS, cells were blocked in fresh bovine serum albumin (BSA; cat. no. AR1006; Boster Biological Technology) buffer $(0.5 \%$ Triton X-100, 2\% BSA, and 0.1\% Tween 20 dissolved in PBS) at room temperature for $1 \mathrm{~h}$, and incubated with the primary antibodies anti-c-Jun/AP-1, anti-Chop (cat. no. GB11024; dilution, 1:100; Servicebio, Inc., Boston, MA, USA) and anti- $\alpha$-SMA, overnight at $4^{\circ} \mathrm{C}$. Cells were treated with 
secondary antibodies (goat anti-mouse $\operatorname{IgG}$ ) and images were obtained as aforementioned.

Terminal deoxynucleotidyl-transferase-mediated dUTP nick end labeling (TUNEL) assay. Apoptotic cells characterized by DNA fragmentation in aortic tissues and MOVAS cells were determined by a TUNEL assay using an In Situ Cell Death Detection kit (cat. no. 11684817910; Roche Applied Science, Penzberg, Germany). First, aortic tissues were fixed as aforementioned, dehydrated, embedded, sectioned (5- $\mu \mathrm{m}$ thickness) and incubated with $0.9 \% \mathrm{NaCl}$ for $10 \mathrm{~min}$ at room temperature. Sections were washed twice with PBS, and then incubated with biotinylated nucleotides and terminal deoxynucleotidyl transferase at $37^{\circ} \mathrm{C}$ for $1 \mathrm{~h}$, followed by incubation with $50 \mu \mathrm{l}$ TUNEL reaction mixture for $1 \mathrm{~h}$ at $37^{\circ} \mathrm{C}$ in a dark and humidified room. Sections were then stained with DAPI $(5 \mu \mathrm{g} / \mathrm{ml})$ at room temperature for $20 \mathrm{~min}$. Following four washes in PBS, samples were mounted using anti-fade mounting medium (cat. no. IH0252; Beijing Leagene Biotech Co., Ltd., Beijing, China) and analyzed under a E2000U confocal microscope (4-7 fields were randomly selected; magnifications, x200 and 400). The staining protocol of MOVAS cells was similar. Light green indicated normal DNA (TUNEL-) and bright green indicated damaged DNA (TUNEL+).

Cell Counting Kit-8(CCK-8) assay. MOVAS cells were seeded in 96-well plates (5,000 cells/well), and cell proliferation was monitored every $24 \mathrm{~h}(24,48$ and $72 \mathrm{~h})$ following treatment with Ang II using the CCK-8 assay (cat. no. CK04; Dojindo Molecular Technologies, Inc., Kumamoto, Japan). Following the manufacturer's protocol, the absorbance was read at $450 \mathrm{~nm}$.

Monolayer wound healing assay. For the monolayer wound healing assay, MOVAS cells $\left(6 \times 10^{5}\right.$ cells/well) were seeded in 6-well plates, and a scratch was introduced on the cell layer after $24 \mathrm{~h}$. A total of three separate wounds were scratched using a $200 \mu \mathrm{l}$ pipette tip, and floating cells and cell debris were washed away with PBS. Fresh medium with $10 \%$ FBS was added, along with $20 \mathrm{nM}$ Ang II for the experimental group, with continued cultured at $37^{\circ} \mathrm{C}$. The wound was imaged at 0 and $24 \mathrm{~h}$, under a transmission microscope (magnification, $\mathrm{x} 100$ ), and the gap distances were measured.

Western blotting assay. Mouse aortas were used for protein extraction with radioimmunoprecipitation assay lysis buffer (cat. no. P0013B; Beyotime Institute of Biotechnology, Haimen, China), following centrifugation at $12,000 \mathrm{xg}$ for $20 \mathrm{~min}$ at $4^{\circ} \mathrm{C}$. Protein concentrations were determined using the bicinchoninic acid assay kit (cat. no. WLA004a; Wanleibio, Co., Ltd., Shanghai, China), according to the manufacturer's protocol. For western blotting, cell lysates ( $35 \mu \mathrm{g}$ per lane) were resolved via $10 \%$ (wt/vol) SDS-PAGE and transferred to a polyvinylidene difluoride membrane (Merck KGaA). The membranes were blocked with $5 \%$ non-fat dry milk in TBS-Tween 20 for $1.5 \mathrm{~h}$ at room temperature, and incubated overnight at $4^{\circ} \mathrm{C}$ with primary antibodies against c-Jun/Ap-1 (cat. no. 711202; dilution, 1:200; Invitrogen; Thermo Fisher Scientific, Inc.), Chop (cat. no. mAb 2895; dilution 1:1,000; Cell Signaling Technology,
Inc., Danvers, MA, USA), GAPDH (cat. no. A00227-1; dilution 1:100; Boster Biological Technology), and $\beta$-actin (cat. no. BM0627; dilution 1:100; Boster Biological Technology). The membranes were washed four times, and incubated with the horseradish peroxidase-conjugated secondary antibody (cat. no. 111-035-003; dilution 1:8,000; Jackson Immuno Research Laboratories, Inc., West Grove, PA, USA) in blocking buffer for $1 \mathrm{~h}$ at room temperature. The bands were visualized using an enhanced chemiluminescence kit (Pierce; Thermo Fisher Scientific, Inc.). ImageJ 1.42q (National Institutes of Health, Bethesda, MD, USA) was used for densitometry analyses.

Reverse transcription-quantitative polymerase chain reaction (RT-qPCR). TRIzol ${ }^{\circledR}$ (cat. no. 10296028; Invitrogen; Thermo Fisher Scientific, Inc.) was used to extract total RNA from MOVAS cells following treatment. RNA samples were reverse-transcribed into cDNA with different primers using the First Strand Synthesis kit (RR036A; Takara Biotechnology Co.,Ltd., Dalian, China), following the manufacturer's protocol. The RT reaction was conducted at $37^{\circ} \mathrm{C}$ for $15 \mathrm{~min}$ and then at $85^{\circ} \mathrm{C}$ for $5 \mathrm{sec}$. cDNA was then stored at $-20^{\circ} \mathrm{C}$ until use. qPCR was performed using SYBR ${ }^{\circledR}$ Premix Ex Taq (RR820A; Takara Biotechnology Co., Ltd.) and an ABI PRISM 7900HT Sequence Detection System (Applied Biosystems; Thermo Fisher Scientific, Inc.). qPCR was performed as follows: $95^{\circ} \mathrm{C}$ for $30 \mathrm{sec}$, and then 40 cycles of $95^{\circ} \mathrm{C}$ for $5 \mathrm{sec}$ and $60^{\circ} \mathrm{C}$ for $30 \mathrm{sec}$. Relative gene expression was determined using the $2^{-\triangle \Delta \mathrm{Cq}}$ method (23). GAPDH was used as an internal reference. The following primers were used: mouse c-Jun, forward 5'-GGGAGCATTTGGAGAGTCCC-3', reverse 5'-TTTGCA AAAGTTCGCTCCCG-3'; mouse Ddit3 forward 5'-CTGCCT TTCACCTTGGAGAC-3', reverse 5'- CGTTTCCTGGGG ATGAGATA-3'; mouse GAPDH, forward 5'-GGGAAATTC AACGGCACAGT-3' and reverse 5'-AGATGGTGATGGGCT TCCC-3'. All PCRs were performed in triplicate. All results were analyzed using SDS Software (version 2.4; Applied Biosystems; Thermo Fisher Scientific, Inc.).

Prediction of c-Jun/Ap-1 binding sites in the mouse Ddit3 promoter. The mouse Ddit3 promoter region sequence (-1.09 $\mathrm{kb}$ to $0 \mathrm{~kb}$ ) was downloaded from the UCSC Genome Browser (http:/genome.ucsc.edu/). Potential transcription factors and binding sites were predicted using the Promoter 2.0 Prediction Server (http://www.cbs.dtu.dk/services/Promoter/) and JASPAR database (http://jaspar.genereg.net/).

Chromatin immunoprecipitation (ChIP) assay. A ChIP kit was purchased from Beyotime Institute of Biotechnology (cat. no. P2078), and ChIP assay was conducted following the manufacturer's protocol. MOVAS cells were cross-linked (40\% methanol solution, $37^{\circ} \mathrm{C}$ for $15 \mathrm{~min}$ ) and then sonicated $\left(0^{\circ} \mathrm{C}, 50 \%\right.$ power with 4 cycles of $5 \mathrm{sec}$ on, $5 \mathrm{sec}$ off $)$ when they reached $90 \%$ confluency in $10 \mathrm{~cm}$ cell culture dishes, followed by IP with a polyclonal anti-c-Jun/Ap-1 antibody (cat. no. 711202; Invitrogen; Thermo Fisher Scientific, Inc.). Normal IgG (cat. no. ab172730; Abcam, Cambridge, UK) was used as a negative control. The supernatant was used as an input control. Precipitated DNA was amplified by PCR using Ddit3-specific primers (forward, 5'-CTGAGTGGCGGATGT 
A
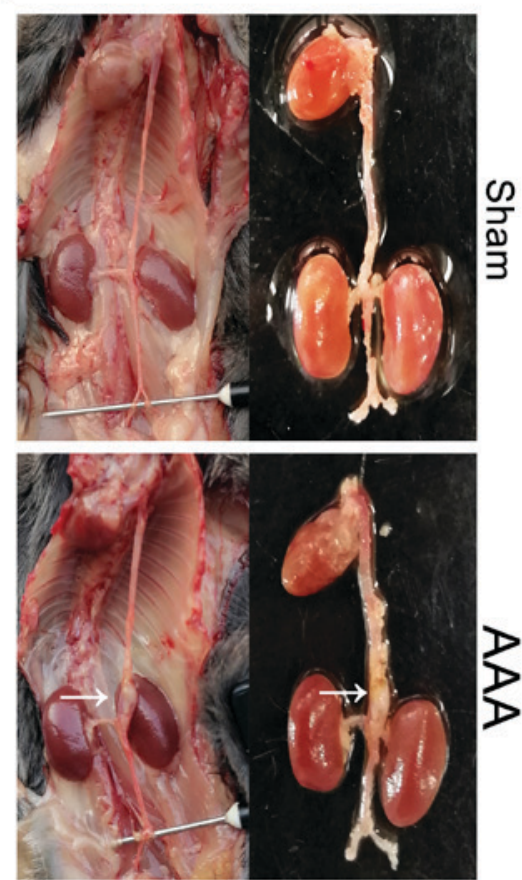

B
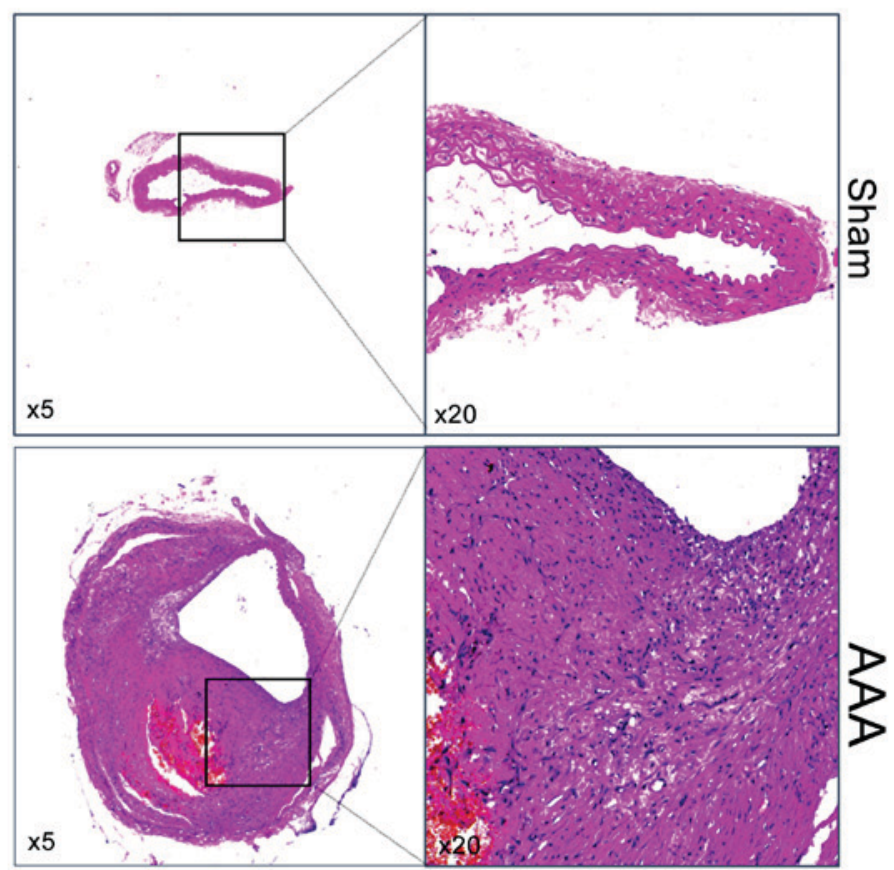

C

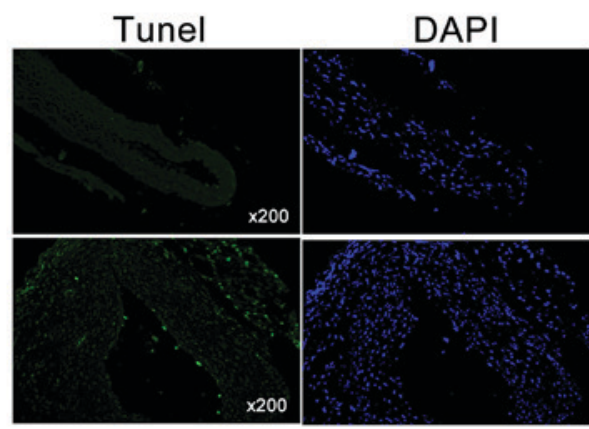

a-SMA

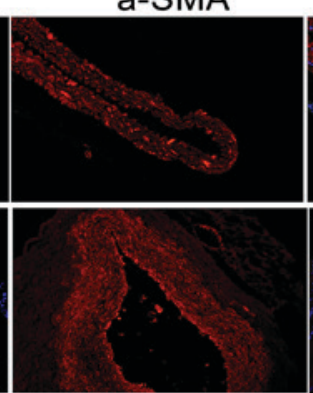

Merged
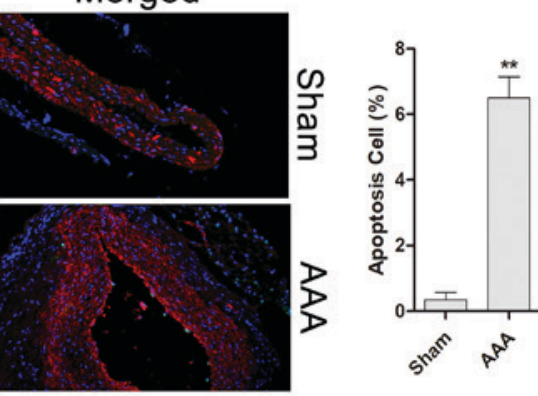

D
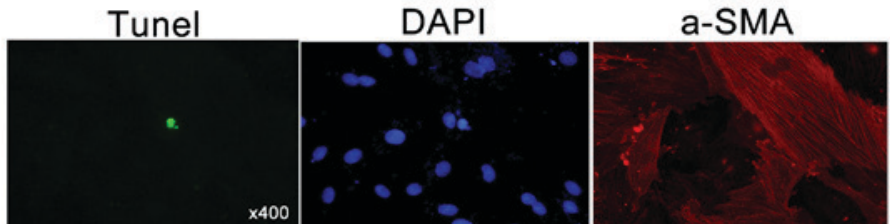

Merged

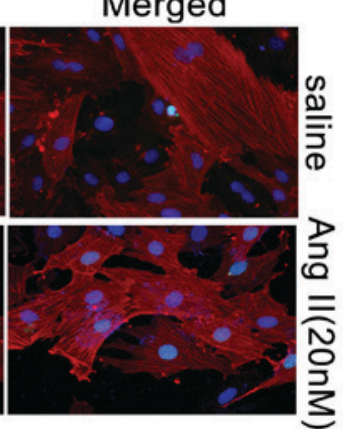

Figure 1. AAA is induced by Ang II in $\mathrm{ApoE}^{-/}$mice. (A) Morphology of Ang II-induced AAA and normal aortas (control) in ApoE ${ }^{-/}$mice; white arrows indicate a typical AAA. (B) Aorta cross-sections stained with hematoxylin and eosin. (C) Representative immunofluorescence and TUNEL staining for the detection of DNA fragmentation in Ang II-induced AAA and saline-treated aortas (magnification, x200). (D) TUNEL assay for detecting cell apoptosis in MOVAS cells treated (or not treated, saline) with Ang II by fluorescence microscopy (magnification, x400). Light green indicates normal DNA (TUNEL-) and bright green indicates damaged DNA (TUNEL+). ${ }^{* *} \mathrm{P}<0.01$ vs. respective Sham. AAA, abdominal aortic aneurysm; Ang II, angiotensin II; ApoE ${ }^{-\digamma}$, apolipoprotein E-deficient; TUNEL, terminal deoxynucleotidyl transferase-mediated dUTP nick end labeling; $\alpha$-SMA, $\alpha$-smooth muscle actin.

AAGGG-3'; reverse, 5'-GGTCCAGGAGCCTACCAATC-3'). PCR products (74 bp) were analyzed by $2 \%$ agarose gel (cat. no. 5261; Takara Biotechnology Co., Ltd.) electrophoresis and visualized using GoldView ${ }^{\mathrm{TM}}$ (cat. no. G8140; Beijing Solarbio Science \& Technology Co., Ltd., Beijing, China) under an ultraviolet light.
Statistical analyses. All results are presented as the mean \pm standard error of the mean from three independent experiments. Statistical differences were evaluated by one-way analysis of variance followed by a Tukey's comparison test. Statistical significance was evaluated using GraphPad Prism (version 5.0; GraphPad Software Inc., La Jolla, CA, USA). 

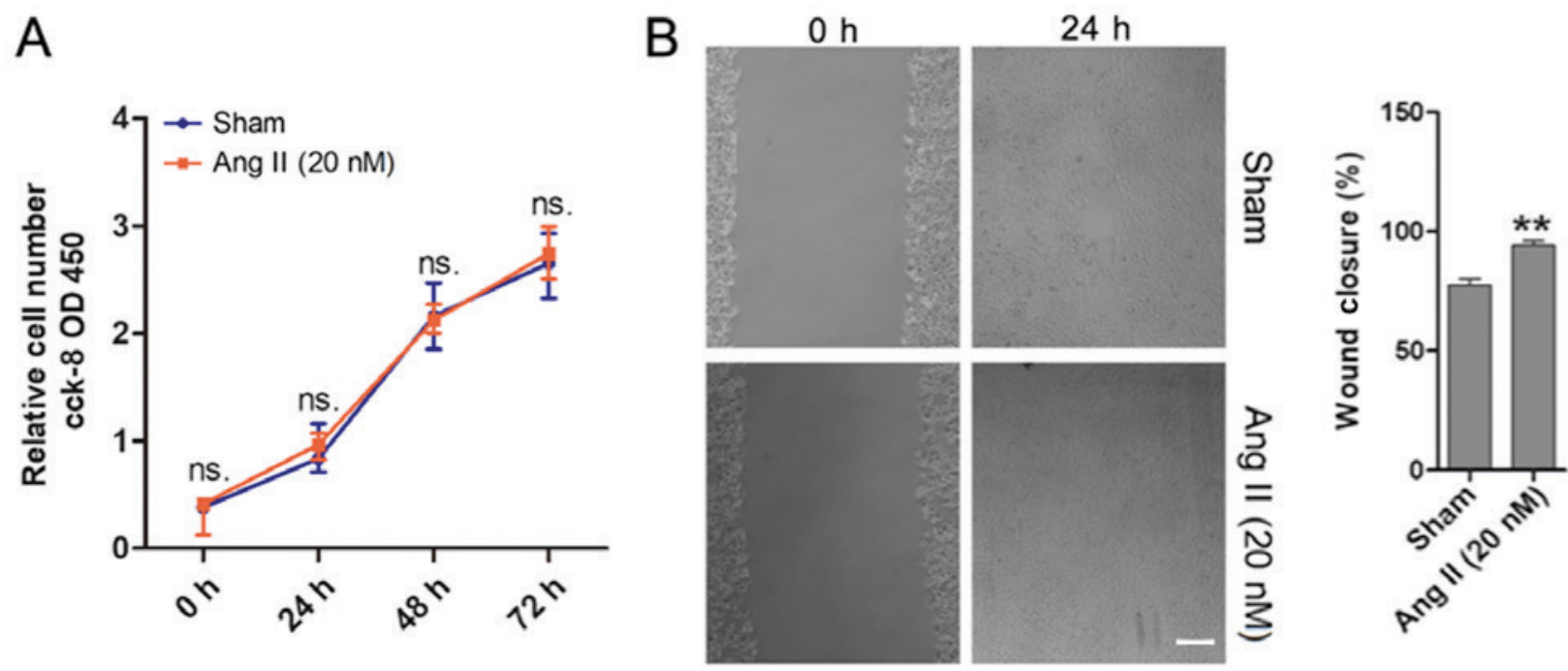

Figure 2. Ang II induces cell migration but does not affect cell proliferation. (A) CCK-8 assay growth curves revealed that Ang II had no effect on the proliferation of MOVAS cells (cells were treated with Ang II $20 \mathrm{nM}$, or were untreated, for 0, 24, 48 and $72 \mathrm{~h}$. (B) The wound-healing assay demonstrated that the migration ability of MOVAS cells was increased following treatment with $20 \mathrm{nM}$ of Ang II. Scale bar, $100 \mathrm{~mm} .{ }^{* *} \mathrm{P}<0.01 \mathrm{vs}$. Sham; $\mathrm{n} \geq 3$. Ang II, angiotensin II; CCK-8, Cell Counting Kit-8; OD450, optical density at $450 \mathrm{~nm}$; ns, non-significant.

$\mathrm{P}<0.05$ was considered to indicate a statistically significant difference.

\section{Results}

Abdominal aortic aneurysm was induced by Ang II in ApoE - $^{-}$ mice. After 4 weeks of Ang II treatment, AAA developed in $\mathrm{ApoE}^{-/-}$mice but not in saline-infused $\mathrm{ApoE}^{-/-}$mice, which exhibited normal aortas (Fig. 1A). Histologically, compared with control aortas, Ang II-induced AAA was characterized by expansion and thickening of the aortic wall, and the cells of the aortic wall exhibited a disordered arrangement (Fig. 1B). Subsequently, apoptosis in Ang II-induced AAA and Ang II-treated MOVAS cells was evaluated by TUNEL staining. Compared with the sham group (saline-treated), the Ang II-treated AAA group exhibited an increase in the percentage of TUNEL+ cells (Fig. 1C). The percentage of apoptotic MOVAS cells with DNA fragmentation under Ang II treatment was significantly higher compared with that for saline-treated cells (Fig. 1D).

Ang II affects MOVAS cell migration in vitro. To investigate the role of Ang II in the cell proliferation and migration of MOVAS cells, CCK-8 and monolayer wound healing assays were performed. The results demonstrated that Ang II did not affect MOVAS cell proliferation (Fig. 2A). However, the monolayer wound healing assay revealed that Ang II promoted MOVAS cell migration (Fig. 2B), and this was in agreement with the results from Greene et al (24).

c-Jun and Chop were simultaneously upregulated in vivo and in vitro in response to Ang II. To investigate the expression of c-Jun during Ang II-induced AAA formation, immunofluorescence was used. c-Jun levels were observed to be higher in Ang II-induced AAA compared with normal aortas (Fig. 3A). Western blotting also demonstrated that Chop was markedly upregulated (Fig. 3B), consistent with the results of a previous study (4). To examine c-Jun and Chop expression levels in MOVAS cells treated with Ang II, cells were co-stained with $\alpha$-SMA and either c-Jun or Chop. c-Jun and Chop expression were increased following treatment with Ang II. Western blotting revealed that c-Jun and Chop protein levels increased in response to Ang II in a dose-dependent manner following treatment with 0-50 nM Ang II; however, following treatment with $100 \mathrm{nM}$ Ang II, the expression levels of c-Jun and Chop markedly decreased compared with $20 \mathrm{nM}$ Ang II (Fig. 3C). These results were confirmed by immunofluorescence, which demonstrated that c-Jun was elevated and was primarily localized in the nuclei of MOVAS cells (Fig. 3D). Furthermore, Chop was also observed to be localized in the nuclei and cytoplasm of MOVAS cells (Fig. 3E).

Chop expression was suppressed by siRNA-mediated c-Jun silencing. Based on the aforementioned results, it was hypothesized that there may be underlying connections between c-Jun/Ap-1 and Ddit3. AP-1 is a transcription factor consisting of a dimer of c-Jun and c-Fos. However, whether Ddit3 is activated by Ap-1 is unknown. Following this line of thought, c-Jun was downregulated using c-Jun-targeted siRNAs and the expression of Chop was assessed. RT-qPCR revealed that c-Jun transcript levels were reduced by $>60 \%$ at $24 \mathrm{~h}$ post-transfection with c-Jun-si2 and c-Jun-si3 [P $<0.001$ vs. negative control (NC)-si], but c-Jun-si1 had no effect $(\mathrm{P}>0.05$ vs. NC-si). GAPDH was used as an internal control (Fig. 4A). Western blotting revealed that the protein levels of c-Jun were also decreased following transfection with c-Jun-si2 and c-Jun-si3 for $48 \mathrm{~h}$ in MOVAS cells ( $\mathrm{P}<0.05$ vs. NC-si) but not with c-Jun-sil ( $\mathrm{P}>0.05$ vs. NC-si), also using GAPDH as an internal control (Fig. 4B). MOVAS cells were transfected with effective siRNAs (c-Jun-si2 and c-Jun-si3) for $24 \mathrm{~h}$, and the transfected cells were incubated with Ang II (20 nM) for $24 \mathrm{~h}$ prior to mRNA or protein assessments. RT-qPCR subsequently demonstrated that the mRNA expression levels of Ddit3 were significantly reduced ( $\mathrm{P}<0.05$ vs. NC-si) (Fig. 4C), and western 


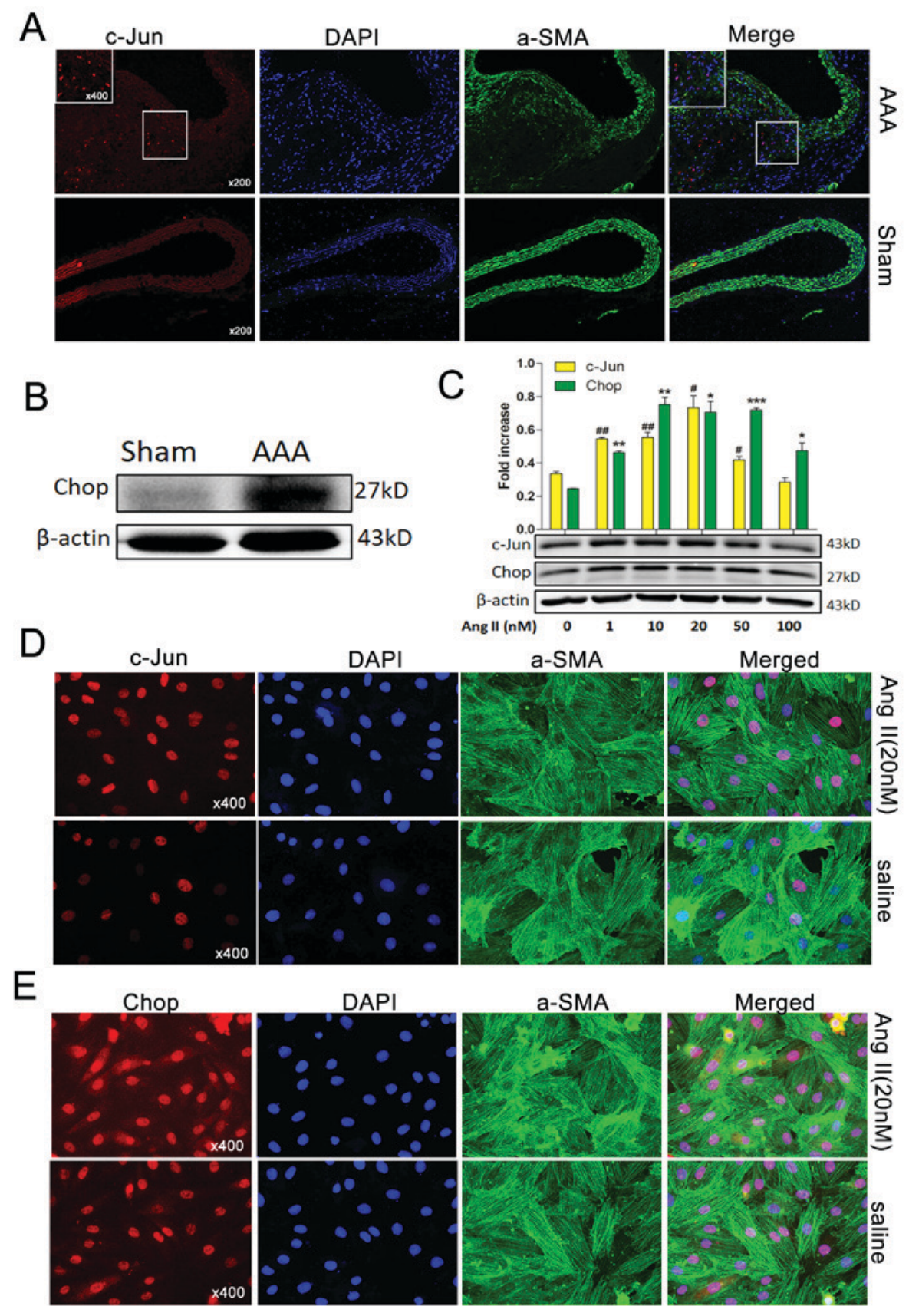

Figure 3. Ap-1 and Chop are induced by Ang II in vivo and in vitro. (A) Immunofluorescence staining with c-Jun/Ap-1 (red) expression and localization in the aortas of Ang II-induced AAA mice and normal aortas. $\alpha$-SMA, green; DAPI, blue. Magnifications, x200 and 400. (B) Western blotting exhibiting Chop expression in normal aortas (control) and Ang II-induced AAA in ApoE ${ }^{-/-}$mice. $\beta$-Actin was used as an internal control. (C) Western blotting demonstrated that c-Jun/Ap-1 and Chop protein levels in MOVAS cells were induced in a dose dependent manner by varying concentrations ( $0,1,10,20$ and $50 \mathrm{nM})$ of Ang II for $36 \mathrm{~h}$. Immunofluorescence staining demonstrating (D) c-Jun/Ap-1 (red) and (E) Chop (red) expression and localization in MOVAS cells induced (or not) by Ang II ( $20 \mathrm{nM}$ ) for $36 \mathrm{~h}$. $\alpha$-SMA (green), DAPI (blue). Magnification, $\mathrm{x} 400$. Results are presented as the mean \pm standard error of the mean; $\mathrm{n}=3 .{ }^{\#} \mathrm{P}<0.05$, ${ }^{*} \mathrm{P}<0.05,{ }^{\# \#} \mathrm{P}<0.01,{ }^{* * *} \mathrm{P}<0.01$ and ${ }^{* * * *} \mathrm{P}<0.001$ vs. Control $(0 \mathrm{nM})$. Ap-1, activator protein 1; Chop, C/EBP homologous protein; Ang II, angiotensin II; $\alpha$-SMA, $\alpha$-smooth muscle actin AAA, abdominal aortic aneurysm; $\mathrm{ApoE}^{-1}$, apolipoprotein E-deficient.

blotting revealed that c-Jun and Chop protein levels were also reduced following transfection with c-Jun-si2 and c-Jun-si3 $(\mathrm{P}<0.05$ vs. NC-si). $\beta$-actin was used as an internal control (Fig. 4D). These results indicated that c-Jun/AP-1 mediates Chop expression in MOVAS cells.

c-Jun/Ap-1 binds to the mouse promoter region in MOVAS cells. To further evaluate the aforementioned hypothesis, the promoter sequence of mouse Ddit3 (encoding Chop) was evaluated using online databases $(-1.09 \mathrm{~kb}$ to $0 \mathrm{~kb})$. The sequence analysis suggested five potential c-Jun/Ap-1 binding sites in the Ddit3 promoter region (Fig. 5A). ChIP assays were performed to further test whether AP-1 binds to the Ddit3 promoter in MOVAS cells. DNA was crushed into fragments (100-250 bp) following sonication and amplified by ChIP-PCR using Ddit3 promoter-specific primers. The DNA fragments pulled down by anti-c-Jun/AP-1 primary antibodies were amplified by Ddit3 promoter-specific primers (Fig. 5B), 
A
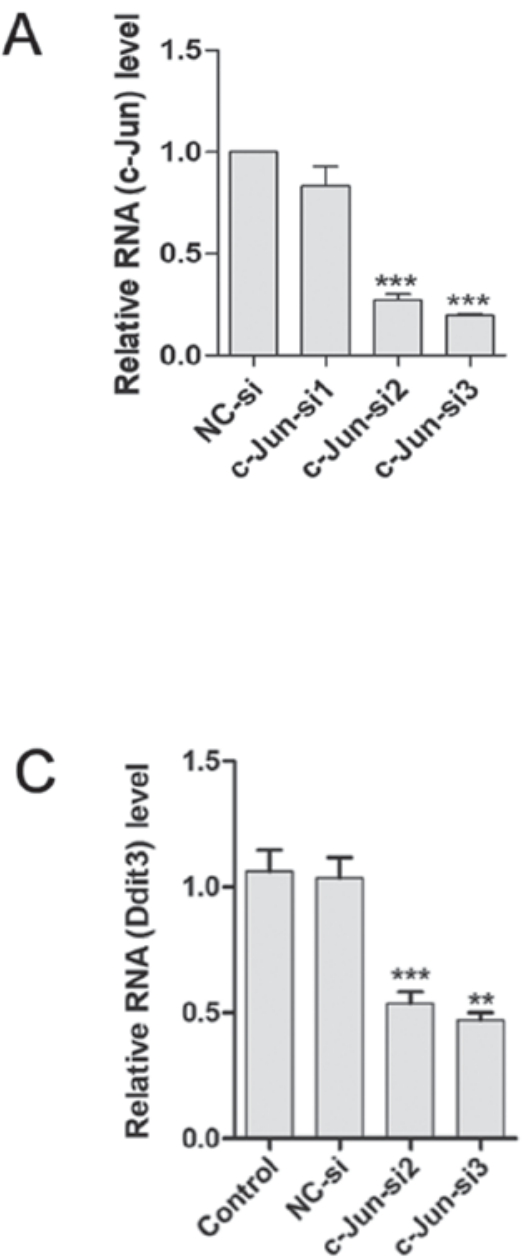
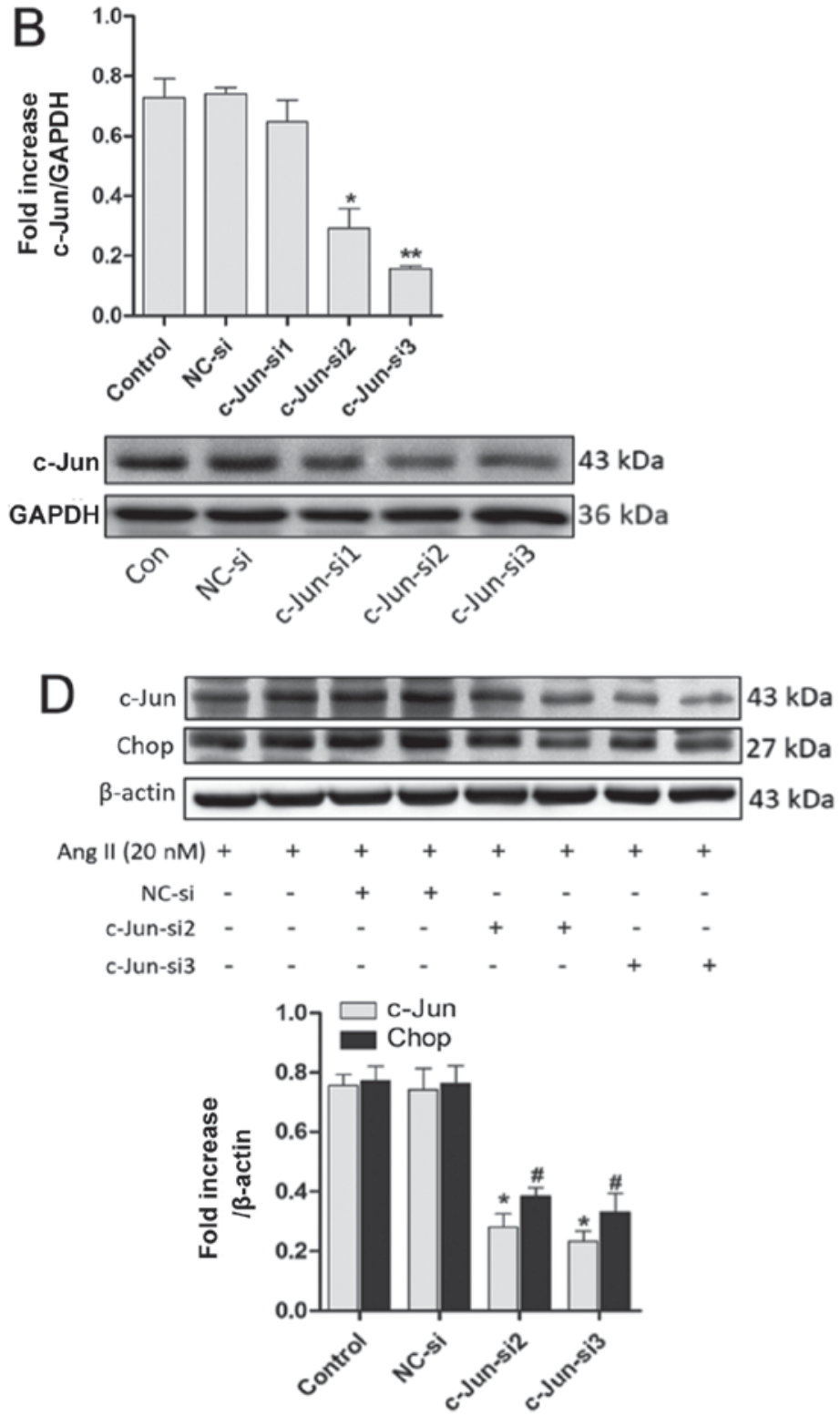

Figure 4. Downregulation of c-Jun/Ap-1 by c-Jun-siRNA suppresses Chop expression. (A) mRNA levels of c-Jun were assessed by RT-qPCR in MOVAS cells transfected with siRNAs for $24 \mathrm{~h}$; GAPDH was used as an internal control ( $\mathrm{n} \geq 3$ ). (B) Western blotting demonstrated the Ap-1 levels in MOVAS cells transfected with siRNAs for $48 \mathrm{~h}$; GAPDH was used as an internal control $(\mathrm{n}=3)$. MOVAS cells were transfected with siRNAs for $24 \mathrm{~h}$ and the transfected cells were treated with Ang II ( $20 \mathrm{nM}$ ) for $24 \mathrm{~h}$. (C) The mRNA expression level of Ddit3 were detected by RT-qPCR; GAPDH was used as an internal control (n $\geq 3$ ), (D) Protein levels were detected by western blotting with anti-c-Jun, anti-Chop, and anti- $\beta$-actin (internal control) antibodies ( $\mathrm{n}=3$ ). All results are presented as the mean \pm standard error of the mean. ${ }^{*} \mathrm{P}<0.05 ;{ }^{*} \mathrm{P}<0.05,{ }^{* *} \mathrm{P}<0.01,{ }^{* * * *} \mathrm{P}<0.001$ vs. NC-si. Ap-1, activator protein 1; Chop, C/EBP homologous protein; RT-qPCR, reverse transcription quantitative-polymerase chain reaction; siRNA, small interfering RNA; Ang II, angiotensin II; NC-si, negative control siRNA; Ddit3, DNA damage-inducible transcript 3 protein.

indicating that c-Jun/Ap-1 binds directly to the Ddit3 promoter and may regulate its expression.

\section{Discussion}

Previous evidence suggests that Ang II serves important roles in cardiovascular homeostasis by directly regulating ER signaling and upregulating Chop expression (4), the latter being involved in a number of diseases, including atherosclerosis (25), hypertension (26) and AAA $(4,27)$. Furthermore, Chop is implicated in the programmed cell death pathway during ER stress $(18,19,28)$, and apoptosis in vascular smooth muscle cells (VSMCs) is associated with
AAA formation, and may contribute to arterial wall thinning, expansion and eventual rupture $(4,27)$. Previous studies have indicated that Ang II may promote ER stress signaling via eukaryotic translation initiation factor $2-\alpha$ kinase 3 and the phospho-eukaryotic translation initiation factor $2 \alpha$-mediated upregulation of cyclic AMP-dependent transcription factor ATF4 (29,30), a transcription factor of Ddit3, and may lead to the expression of Chop. Consistent with these observations, Ang II was also demonstrated to stimulate intracellular reactive oxygen species generation $(14,31)$, and to upregulate c-Jun/AP-1 expression by activating the mitogen-activated protein kinase-JNK pathway (32). Therefore, it was hypothesized that c-Jun/AP-1 may cause Chop overexpression and 

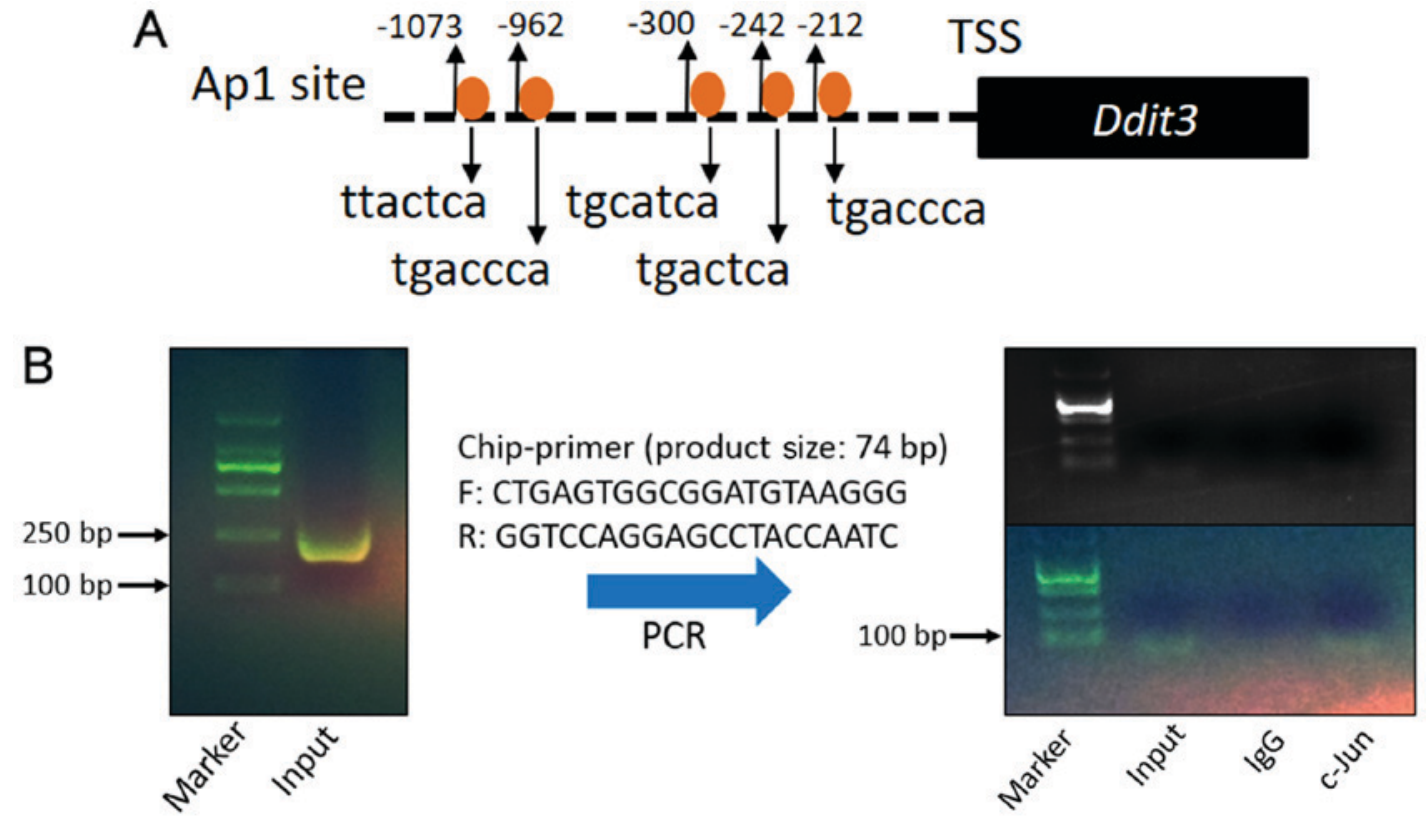

Figure 5. c-Jun/Ap-1 binds to the mouse Ddit3 promoter region in MOVAS cells. (A) Sequence of the mouse Ddit3 promoter region (-1090 to 0) and sketch of putative AP-1 binding sites. (B) Binding of Ap-1 to the mouse Ddit3 promoter was detected via ChIP-PCR using Ddit3 promoter-specific primers. An anti-Ap-1 antibody was used for IP in ChIP assays, and anti-rabbit IgG was used for control experiments. DNA fragments were obtained after cell cross-linking and sonication. Ap-1, activator protein 1; Ddit3, DNA damage-inducible transcript 3 protein; ChIP, chromatin immunoprecipitation; PCR, polymerase chain reaction; IgG, immunoglobulin G; TSS, transcription start sequence.

may indirectly induce apoptosis, thus accelerating the development of AAA.

A number of AAA induction methods in animal models has been developed, and three approaches are frequently used by researchers, including the porcine pancreatic elastase model (33), the calcium chloride model (34) and the Ang II model (35). Notably, Ang II induced AAA in $\mathrm{ApoE}^{-/-}$mice is the most widely used AAA animal model among the three methods, and it has a number of features similar to the human AAA disease, including progressive abdominal aorta dilation, diffuse inflammation, VSMC apoptosis and extracellular matrix deformation (36). In the present study this latter model was adopted, and $\mathrm{ApoE}^{-/-}$mice were treated with Ang II (1,000 ng/kg/min) for 4 weeks (24). Based on the adverse effects encountered in a previous study (4), 30 ApoE $^{-/-}$mice were randomly and equally assigned to Ang II or saline treatment groups, which were delivered via osmotic minipumps. In vivo, apoptosis was induced in the Ang II-induced AAA model, and the TUNEL-positive signal seemed to be localized in the adventitia, since AAA involves structural deterioration of the tissue architecture, leading to a progressively enlarged abdominal aorta. Concurrently, the tunica media became loose, and resident VSMCs and infiltrating macrophages have been demonstrated to release matrix metalloproteinases (37), which increase VSMC migration from the tunica media to the adventitia, which may explain the large number of VSMCs observed in the adventitia following AAA. Furthermore, the migration ability and apoptosis of MOVAS cells treated with Ang II were increased. Thus, combining in vivo and in vitro data, it may be that increased VSMC apoptosis and migration lead to $\mathrm{AAA}(38,39)$.
c-Jun/AP-1 and Chop were also observed to be upregulated in Ang II-induced AAA and Ang II-treated MOVAS cells. Additionally, the present results demonstrated that the increase in c-Jun and Chop expression levels in response to Ang II was dose-dependent, with c-Jun and Chop expression levels decreasing at higher doses $(100 \mathrm{nM})$. A possible interpretation is that Ang II may induce ER stress, intracellular reactive oxygen species generation and inflammation, leading to cell apoptosis. A previous study demonstrated that VSMC cell apoptosis was directly dependent on Ang II concentration, and that $50 \mathrm{nM}$ Ang II may induce 25-30\% cell apoptosis; $100 \mathrm{nM}$ Ang II led to cell death without activation of an apoptotic pathway (4). In addition, the present study indicated that Chop was suppressed when c-Jun was silenced. A previous study revealed that Ang II may generate a consistent and substantial amount of reactive oxygen species, leading to a long-lasting activation of AP-1 (14). Activated AP-1 directly controls gene expression and indirectly regulates cell functions, including cell proliferation, migration, differentiation and apoptosis $(8,9)$. The present results revealed that AP-1 may be involved in the expression of Chop, and may therefore affect cell apoptosis and aortic tissue remodeling. Furthermore, a ChIP assay demonstrated that c-Jun/Ap-1 may bind to a consensus sequence of the mouse Ddit3 promoter region, suggesting that c-Jun/AP-1 may be a transcriptional regulator of Ddit3. In addition, a previous study indicated that the selective inhibition of JNK not only prevented the development of AAA, but also caused its regression in Ang II-induced AAA models by ameliorating the extracellular matrix metabolism and enhancing aortic tissue repair (5). Unfortunately, the link between this amelioration and Chop expression was not examined. 
The present work had certain limitations. Firstly, the focus of the study was to determine whether c-Jun/AP-1 may mediate Chop expression. In the in vitro assays the impact of ATF4 signaling on ER stress was not assessed; siRNA-mediated downregulation of ATF4 would have been useful as a negative control for the evaluation of Chop expression and for controlling the impact of ER stress. Secondly, to further evaluate the hypothesis that c-Jun/AP-1 binds to the proximal promoter of the Ddit3 gene in MOVAS cells increasing Chop expression, the upregulation of c-Jun, in addition to its downregulation, could have been evaluated with respect to Chop expression. Ang II-treated and c-Jun-overexpressing cells could have been analyzed with respect to c-Jun binding to the Ddit 3 promoter. Thirdly, in order to further prove that Ap-1 is a Ddit3 transcription factor, more group comparisons of ChIP assays are required. c-Jun knockdown and/or overexpression may confirm whether Ap-1 is a transcription factor of Ddit3, and whether treatment with Ang II promotes Ap-1 binding to the Ddit3 promoter in MOVAS cells. Thus, additional studies are required to determine the precise role of c-Jun/AP-1 in AAA, and to confirm whether c-Jun/AP-1 causes Chop overexpression, thereby accelerating AAA development.

In conclusion, the present study demonstrated that c-Jun/AP-1 was overexpressed in an Ang II-induced AAA model and in Ang II-treated MOVAS cells, and that it mediated the expression of Chop. Therefore, c-Jun/AP-1 may be a novel target for AAA therapy.

\section{Acknowledgements}

Not applicable.

\section{Funding}

This study was funded by research grants from The National Natural Science Foundation of China (grant no. 81370417) and Hubei Natural Science Foundation Project (grant no. 2018CFB465).

\section{Availability of data and materials}

All data generated or analyzed during the present study are included in this published article.

\section{Authors' contributions}

JY and MZ were involved in the conception and supervision of the project. JY and DS were involved in the design of the study. DS performed the experiments. DS, YL and JQ analyzed the results. DS and SM performed and analyzed the CCK-8 and migration assays. DS, YL and JQ prepared the paper. All the authors read and approved the final manuscript.

\section{Ethics approval and consent to participate}

All animal experiments were performed in accordance with the Animal Research Reporting of in vivo Experiments and the National Institutes of Health guidelines for animal welfare, and the study was approved by the Animal Research Committee of Tongji Medical College (Wuhan, China).

\section{Patient consent for publication}

Not applicable.

\section{Competing interests}

The authors declare that they have no competing interests.

\section{References}

1. Wassef M, Upchurch GR Jr, Kuivaniemi H, Thompson RW and Tilson MD III: Challenges and opportunities in abdominal aortic aneurysm research. J Vasc Surg 45: 192-198, 2007.

2. Lu H, Rateri DL, Bruemmer D, Cassis LA and Daugherty A: Novel mechanisms of abdominal aortic aneurysms. Curr Atheroscler Rep 14: 402-412, 2012.

3. Svensjö S, Björck M and Wanhainen A: Editor's choice: Five-year outcomes in men screened for abdominal aortic aneurysm at 65 years of age: A population-based cohort study. Eur J Vasc Endovasc Surg 47: 37-44, 2014.

4. Li Y, Lu G, Sun D, Zuo H, Wang DW and Yan J: Inhibition of endoplasmic reticulum stress signaling pathway: A new mechanism of statins to suppress the development of abdominal aortic aneurysm. PLoS One 12: e0174821, 2017.

5. Yoshimura K, Aoki H, Ikeda Y, Fujii K, Akiyama N, Furutani A, Hoshii Y, Tanaka N, Ricci R, Ishihara T, et al: Regression of abdominal aortic aneurysm by inhibition of c-Jun N-terminal kinase. Nat Med 11: 1330-1338, 2005.

6. Kristensen KE, Torp-Pedersen C, Gislason GH, Egfjord M, Rasmussen HB and Hansen PR: Angiotensin-converting enzyme inhibitors and angiotensin II receptor blockers in patients with abdominal aortic aneurysms: Nation-wide cohort study. Arterioscler Thromb Vasc Biol 35: 733-740, 2015.

7. Kent KC: Clinical practice. Abdominal aortic aneurysms. N Engl J Med 371: 2101-2108, 2014.

8. Shaulian E and Karin M: AP-1 as a regulator of cell life and death. Nat Cell Biol 4: E131-E136, 2002.

9. Wisdom R, Johnson RS and Moore C: c-Jun regulates cell cycle progression and apoptosis by distinct mechanisms. EMBO J 18: 188-197, 1999.

10. Shaulian E: AP-1-The Jun proteins: Oncogenes or tumor suppressors in disguise? Cell Signal 22: 894-899, 2010.

11. Hess J, Angel P and Schorpp-Kistner M: AP-1 subunits: Quarrel and harmony among siblings. J Cell Sci 117: 5965-5973, 2004.

12. Chinenov Y and Kerppola TK: Close encounters of many kinds: Fos-Jun interactions that mediate transcription regulatory specificity. Oncogene 20: 2438-2452, 2001.

13. Plotnikov A, Zehorai E, Procaccia S and Seger R: The MAPK cascades: Signaling components, nuclear roles and mechanisms of nuclear translocation. Biochim Biophys Acta 1813: 1619-1633, 2011.

14. Wu S, Gao J, Ohlemeyer C, Roos D, Niessen H, Köttgen E and Gessner R: Activation of AP-1 through reactive oxygen species by angiotensin II in rat cardiomyocytes. Free Radic Biol Med 39: 1601-1610, 2005.

15. Frank GD, Eguchi S, Yamakawa T, Tanaka S, Inagami T and Motley ED: Involvement of reactive oxygen species in the activation of tyrosine kinase and extracellular signal-regulated kinase by angiotensin II. Endocrinology 141: 3120-3126, 2000.

16. Friedman AD: GADD153/CHOP, a DNA damage-inducible protein, reduced CAAT/enhancer binding protein activities and increased apoptosis in 32D c13 myeloid cells. Cancer Res 56: 3250-3256, 1996.

17. Hotamisligil GS: Endoplasmic reticulum stress and atherosclerosis. Nat Med 16: 396-399, 2010.

18. Kim I, Xu W and Reed JC: Cell death and endoplasmic reticulum stress: Disease relevance and therapeutic opportunities. Nat Rev Drug Discov 7: 1013-1030, 2008.

19. Oyadomari S and Mori M: Roles of CHOP/GADD153 in endoplasmic reticulum stress. Cell Death Differ 11: 381-389, 2004

20. Ubeda M, Vallejo M and Habener JF: CHOP enhancement of gene transcription by interactions with Jun/Fos AP-1 complex proteins. Mol Cell Biol 19: 7589-7599, 1999.

21. Guide for the Care and Use of Laboratory Animals. NIH Publication No. 85-23. National Institutes of Health, Bethesda, MD, 1996. 
22. Daugherty A, Manning MW and Cassis LA: Angiotensin II promotes atherosclerotic lesions and aneurysms in apolipoprotein E-deficient mice. J Clin Invest 105: 1605-1612, 2000.

23. Livak KJ and Schmittgen TD: Analysis of relative gene expression data using real-time quantitative PCR and the 2(-Delta Delta C(T)) method. Methods 25: 402-408, 2001.

24. Greene EL, Lu G, Zhang D and Egan BM: Signaling events mediating the additive effects of oleic acid and angiotensin II on vascular smooth muscle cell migration. Hypertension 37 308-312, 2001

25. Cominacini L, Garbin U, Mozzini C, Stranieri C, Pasini A, Solani E, Tinelli IA and Pasini AF: The atherosclerotic plaque vulnerability: Focus on the oxidative and endoplasmic reticulum stress in orchestrating the macrophage apoptosis in the formation of the necrotic core. Curr Med Chem 22: 1565-1572, 2015.

26. Sun Y, Zhang T, Li L and Wang J: Induction of apoptosis by hypertension via endoplasmic reticulum stress. Kidney Blood Press Res 40: 41-51,2015.

27. Qin Y, Wang Y, Liu O, Jia L, Fang W, Du J and Wei Y: Tauroursodeoxycholic acid attenuates angiotensin ii induced abdominal aortic aneurysm formation in apolipoprotein E-deficient mice by inhibiting endoplasmic reticulum stress. Eur J Vasc Endovasc Surg 53: 337-345, 2017.

28. Zinszner H, Kuroda M, Wang X, Batchvarova N, Lightfoot RT, Remotti H, Stevens JL and Ron D: CHOP is implicated in programmed cell death in response to impaired function of the endoplasmic reticulum. Genes Dev 12: 982-995, 1998.

29. Harding HP, Novoa I, Zhang Y, Zeng H, Wek R, Schapira M and Ron D: Regulated translation initiation controls stress-induced gene expression in mammalian cells. Mol Cell 6: 1099-1108, 2000

30. Vattem KM and Wek RC: Reinitiation involving upstream ORFs regulates ATF4 mRNA translation in mammalian cells. Proc Natl Acad Sci USA 101: 11269-11274, 2004.

31. Dai DF, Johnson SC, Villarin JJ, Chin MT, Nieves-Cintrón M, Chen T, Marcinek DJ, Dorn GW II, Kang YJ, Prolla TA, et al: Mitochondrial oxidative stress mediates angiotensin II-induced cardiac hypertrophy and Galphaq overexpression-induced heart failure. Circ Res 108: 837-846, 2011.

32. Chiu CZ, Wang BW and Shyu KG: Angiotensin II and the JNK pathway mediate urotensin II expression in response to hypoxia in rat cardiomyocytes. J Endocrinol 220: 233-246, 2014.
33. Pyo R, Lee JK, Shipley JM, Curci JA, Mao D, Ziporin SJ, Ennis TL, Shapiro SD, Senior RM and Thompson RW: Targeted gene disruption of matrix metalloproteinase-9 (gelatinase B) suppresses development of experimental abdominal aortic aneurysms. J Clin Invest 105: 1641-1649, 2000.

34. Bi Y, Zhong H, Xu K, Zhang Z, Qi X, Xia Y and Ren L: Development of a novel rabbit model of abdominal aortic aneurysm via a combination of periaortic calcium chloride and elastase incubation. PLoS One 8: e68476, 2013.

35. Ghoshal S and Loftin CD: Cyclooxygenase- 2 inhibition attenuates abdominal aortic aneurysm progression in hyperlipidemic mice. PLoS One 7: e44369, 2012

36. Trachet B, Fraga-Silva RA, Piersigilli A, Tedgui A, Sordet-Dessimoz J, Astolfo A, Van der Donckt C, Modregger P, Stampanoni MF, Segers P and Stergiopulos N: Dissecting abdominal aortic aneurysm in Ang II-infused mice: Suprarenal branch ruptures and apparent luminal dilatation. Cardiovase Res 105: 213-222, 2015.

37. Browatzki M, Larsen D, Pfeiffer CA, Gehrke SG, Schmidt J, Kranzhofer A, Katus HA and Kranzhofer R: Angiotensin II stimulates matrix metalloproteinase secretion in human vascular smooth muscle cells via nuclear factor-kappaB and activator protein 1 in a redox-sensitive manner. J Vasc Res 42: 415-423, 2005.

38. Schubl S, Tsai S, Ryer EJ, Wang C, Hu J, Kent KC and Liu B: Upregulation of protein kinase cdelta in vascular smooth muscle cells promotes inflammation in abdominal aortic aneurysm. J Surg Res 153: 181-187, 2009.

39. Cai Z, Zhao G, Yan J, Liu W, Feng W, Ma B, Yang L, Wang JA, Tu L and Wang DW: CYP2J2 overexpression increases EETs and protects against angiotensin II-induced abdominal aortic aneurysm in mice. J Lipid Res 54: 1448-1456, 2013.

This work is licensed under a Creative Commons Attribution-NonCommercial-NoDerivatives 4.0 International (CC BY-NC-ND 4.0) License. 